\title{
La Tarea Integrada: Una Forma De Aprender Desde la Mirada Del Uso De Wikis Colaborativas
}

\author{
Integrated task: a way of learning from the view of the use of collaborative wikis
}

\author{
Rossana Fiorella Felipa-Andrade ${ }^{1}$
}

RESUMEN

\begin{abstract}
A lo largo del tiempo, los paradigmas del aprendizaje han evolucionado y han registrado aportes significativos en materia educativa. En este avance, y con el surgimiento de nuevas formas de aprender asociadas a la aparición de las tecnologías de comunicación e información, educadores y estudiantes enfrentan grandes retos al momento de enseñar y aprender. En el presente artículo, se propone revisar el modelo instruccional de David Merrill, enfocado en la tarea integrada, y en sus principios básicos para la concreción de aprendizajes sólidos y funcionales, en directa adhesión con la utilización pertinente de wikis colaborativas en entornos virtuales de aprendizaje.
\end{abstract}

Palabras claves: aprendizaje, tecnología, tarea integrada, wiki.

Abstract

Over time, learning paradigms have evolved and have registered significant contributions in education. In this advance, and with the emergence of new ways of learning associate with emergence of communication and information technologies, educators and students face great challenges when teaching and learning. In this article, it is proposed to review the instructional model of David Merrill, focused on the integrated task, and on its basic principles for the realization of solid and functional learning, in direct adherence to the relevant use of collaborative wikis in virtual learning environments.

Key words: learning, technology, integrated task, wiki

\section{INTRODUCCIÓN}

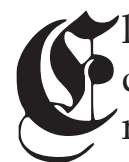
aprendizaje es un fenómeno complejo desde toda perspectiva. Si se le quiere mirar desde el rol del estudiante, es

1 Magíster en Gestión Educativa y docente del Departamento de Humanidades, Universidad Privada del Norte, Lima, Perú. posible encontrar distintos perfiles y mil $y$ un formas de adquirir conocimientos $y$ habilidades que sean útiles para la vida. Si te toma la posición del docente, se halla una gran tarea en la comprensión y en el manejo de los estilos de aprendizaje de una comunidad estudiantil heterogénea con expectativas variadas.

Si a lo anterior se le añade un reto paralelo relacionado a la aparición e intervención de las tecnologías de la comunicación y de la información (TIC), el proceso académico podría tornarse laberíntico y muy espinoso para los actores principales en este escenario. Por tanto, y frente a estas implicancias, se recoge los aportes más reveladores en torno al estudio de los paradigmas del aprendizaje en concordancia con las tendencias y nuevas apariciones en el campo educativo, todas estas vinculadas al uso de tecnologías funcionales y pertinentes en el camino de enseñar y aprender. En esta ruta, es inevitable mencionar la contribución del conductismo, modelo que propone al estudiante como un agente pasivo condicionado permanentemente a los objetivos de aprendizaje establecidos por el docente.1 De igual forma, es necesario recoger lo expuesto por el cognitivismo, cuyo enfoque se respalda en la construcción de esquemas de 
contenido, a partir del procesamiento activo de información. Por su parte, el constructivismo apoya la construcción del aprendizaje junto con la intervención de otros procesos implicados, como la motivación, el involucramiento con la tarea y la autoconfianza. Finalmente, y en la línea de esta propuesta, se analiza los aportes del conectivismo, como una tendencia asociada al aprendizaje que recoge lo postulado por los paradigmas anteriores en función del uso de redes de información y del manejo de herramientas vigentes y pragmáticas en entornos en línea.

Lo comentado hasta aquí permite plantear una interesante integración entre uno de los aportes más importantes en términos de aprendizaje, por David Merrill, quien destaca en su modelo instruccional la proposición de tareas (o actividades) integradas y reguladas por principios altamente eficaces, y el uso acertado de herramientas tecnológicas actuales para afrontar las interminables demandas del binomio aprendizajetecnología, cuya presencia en las aulas puede resultar favorecedora y atractiva para quienes aprenden y enseñan en estos días.

\section{IDEAS GENERALES SOBRE EL APRENDIZAJE}

El términoaprendizajeha sidoy es ampliamente estudiado en distintas áreas académicas, entre las que destacan la psicología y la pedagogía. De esta manera, en las últimas décadas, se han desarrollado diversas investigaciones y revisiones teóricas sobre su concepción y evolución. Un primer acercamiento empírico a la esencia del concepto se sostiene en la idea de un proceso que requiere la actuación activa del sujeto protagonista, quien es el encargado principal de la construcción de su conocimiento y que involucra la noción de un compromiso y vínculo afectivo con lo que se aprende. En palabras de Rodríguez2, "el aprendizaje es por sí mismo un concepto y un proceso, que no tan sólo es individual, sino social y temporal, además de que como concepto es complejo de describir. Por otrolado, López y López3 señalan que "el aprendizaje es un complejo proceso que comprende diversas fases y en el que intervienen multitud de variables personales y contextuales interconexionadas". Por su parte, Leiva4 afirma que "aprender requiere el desarrollo de nuevas acciones o la modificación de las presentes. El aprendizaje es inferencial; es decir, no lo observamos directamente, sino el producto o comportamiento. Observamos el aprendizaje en las expresiones verbales, los escritos y la conducta de la gente".

Hasta este punto, es claro que varios autores intentan definir al aprendizaje como un proceso complejo con distintas implicancias, entre las que destacan el desenvolvimiento social, el cumplimiento de determinadas etapas y un cambio de comportamiento. En cualquier caso, es posible afirmar a que se trata de un ciclo inherente al ser humano, que lo involucra de manera directa y que repercute, en alguna medida, en su conducta frente a lo que se aprende. Ahora bien, si se pretende hacer una referencia a la relación del aprendizaje con las condiciones del mundo contemporáneo, habría que mencionar, ineludiblemente, la presencia de la tecnología y su impacto en las formas de adquirir nuevos conocimientos. En este sentido, Ovelar, Benito y Romo5 agregan que "se ha apreciado una creciente preferencia por el aprendizaje basado en la experiencia, la capacidad de simultanear distintas tareas y el uso intensivo de las TIC como plataforma de colaboración y acceso a la información". En consecuencia, hoy es inevitable estudiar el aprendizaje sin aludir a la intervención de algunas herramientas tecnológicas en la vida académica y personal. 


\section{EVOLUCIÓN DE LOS PARADIGMAS DEL APRENDIZAJE}

Luego de haber revisado algunas concepciones generales sobre el aprendizaje en el apartado anterior, se considera importante retomar, brevemente, algunos de los paradigmas del aprendizaje más relevantes, estudiados a lo largo del siglo XX por investigadores reconocidos a nivel mundial y que han concretado grandes contribuciones en su estudio. En primer lugar, se cita al paradigma conductista, el cual postula, puntualmente, que el estudiante es un receptor pasivo que actúa conforme se desarrollan los factores ambientales. Así, se trata de un sujeto que permanece atento a los objetivos planteados, a las condiciones expresas para desarrollar una actividad determinada. Asimismo, identifica las conductas que el docente espera de él. En ningún caso, debe recurrir a sus conocimientos previos, ni a los esquemas obtenidos de experiencias anteriores. De forma simultánea, el docente es un experto planificador de la secuencia didáctica, los objetivos de aprendizaje y de los recursos que se utilizarán en la sesión. Además, incorpora estrategias para estimular la conducta esperada en sus alumnos, a partir del ofrecimiento de premios o recompensas. (motivación extrínseca). Es "un ingeniero educacional y un administrador de contingencias".6 Como se aprecia, el conductismo elevó grandes esfuerzos en demostrar que la promoción y la concreción de determinadas conductas resultan favorecedores del aprendizaje humano.

En segundo término, se hace mención del paradigma cognitivista, cuyo principio esencial está orientado a la participación dinámica del educando en los procesos académicos que experimenta. "El alumno es entendido como un sujeto activo procesador de información".1 Desde la perspectiva del paradigma cognitivista, el estudiante posee esquemas y representaciones mentales que utiliza en la aplicación de la actividad. Esta información resulta esencial en su proceso de aprendizaje. Por otro lado, con relación al rol del docente, este se encarga de propiciar espacios en los que los estudiantes acuden a los esquemas y representaciones ya existentes. Incluso, los ayuda a discriminar en cuanto a su relevancia y pertinencia para la tarea en cuestión. Además, incentiva la participación y deja de lado el típico y tradicional rol protagónico en la enseñanza. En este punto, la retroalimentación que brinda a los alumnos es trascendente, pues observa de cerca el proceso y su orientación resulta pertinente.

Punto y aparte, en cuanto al paradigma constructivista, la teoría piagetiana sostiene que el alumno cumple un rol activo en la adquisición del conocimiento. En el aula de clase, es el protagonista del proceso, pues desarrolla actividades, a partir de la exploración, la toma de decisiones y la autoconfianza. Asimismo, se activan esquemas de conocimiento que el estudiante involucra en su proceso de aprendizaje. Posteriormente, se acude a la reflexión sobre el significado y la funcionalidad del conocimiento adquirido. En este escenario, el otro agente protagonista, el docente, se vuelve un facilitador y aplica la 'enseñanza indirecta', por cuanto promueve espacios en los que los estudiantes se desenvuelven de manera autónoma, exploran en sus conocimientos previos y reconocen la relevancia del proceso que experimenta. Como bien señala Hernández1, "el maestro es un promotor del desarrollo y de la autonomía de los educandos".

“El constructivismo ha tenido un impacto sustancial en la optimización de las condiciones para el aprendizaje y en el diseño de estrategias instruccionales que apoyen la construcción del conocimiento".7 De esta 
forma, el estudiante puede verse involucrado en procesos académicos complejos:

- Propuesta de actividades auténticas

- Participación activa del educando en los procesos académicos

- Desarrollo de entornos colaborativos para el intercambio de ideas

- Orientación al estudiante para la definición de metas personales

- Guía para la autorregulación en el aprendizaje

- Reflexión sobre las formas de aprendizaje (metacognición)

Anteriormente, se hizo referencia a la presencia de las tecnologías de comunicación e información (TIC) en los procesos de aprendizaje. De esta manera, el paradigma conectivista se centra en la producción de conocimiento e información en entornos virtuales que involucran el uso de herramientas tecnológicas y una interacción social entre los individuos que intercambiar contenidos diversos. Tal como lo menciona Siemens, como se citó en Zapata ", "es una interpretación de algunos de los procesos que se producen en el seno de la Sociedad de la Información y del Conocimiento (SIC), relacionados con la educación, en la que se atribuye un significado y una proyección de estos cambios en el ámbito de la práctica educativa y de su organización". En efecto, el impacto es innegable y la gestión de estos recursos repercuten en los procesos de enseñanza-aprendizaje actuales a partir de dos principios claves:

- El aprendizaje se concreta en conocimientos aplicados en distintos contextos y se encuentran almacenados en diversos dispositivos.

- El individuo debe desarrollar competencias y habilidades para gestionar contenidos en diferentes situaciones, según sus necesidades.
Para terminar con esta revisión, Siemens enuncia que el conectivismo ha de considerarse una teoría, en la medida que toma al aprendizaje "como una extensión del aprendizaje ya existente". ${ }^{8}$ Es seguro que la polémica sobre cómo debe tomarse este enfoque en el ámbito de la investigación no va a concluir pronto. Sin embargo, resulta enriquecedor tomar esta propuesta desde la perspectiva positiva, a partir del planteamiento de nuevos ejes de investigación que favorezcan el entendimiento del aprendizaje.

Durante muchas décadas, se han desarrollado distintos enfoques en cuanto al aprendizaje se refiere. Los paradigmas estudiados (conductista, cognitivista, constructivista y ahora, el conectivista) tienen una esencia diferenciada según los fines que se persiguen. Al respecto, todos estos enfoques persisten hasta nuestros días en poca o buena medida en los procesos de enseñanza-aprendizaje, ya sea en las escuelas, en las instituciones de educación superior o e incluso en el hogar, cuando se trata de formar a nuestros hijos.

Tanto el conocimiento, la habilidad y la actitud son elementos necesarios para el aprendizaje significativo, a pesar de que "el empleo del término competencias ha dado origen a un lenguaje muy amplio en el terreno de la educación". ${ }^{9}$ Más allá de cuánto se ha abordado el famoso enfoque por competencias en los últimos años, estos factores se integran en una unidad que podría responder funcionalmente a las demandas de las sociedades contemporáneas; por lo cual, se ve necesario desarrollarlas y potencializarlas en las aulas de clase.

Tómese este cuadro como un breve consolidado de los principales paradigmas relacionados al aprendizaje y como una propuesta para continuar en el estudio del conectivismo como 
Tabla 1: Evolución de las perspectivas del aprendizaje

\begin{tabular}{|c|c|c|c|}
\hline Conductismo & Cognitivismo & Constructivismo & Conectivismo \\
\hline $\begin{array}{l}\text { Enfoque que propone la } \\
\text { actuación del estudiante a } \\
\text { partir de un comportamiento } \\
\text { pasivo y que se encuentra } \\
\text { condicionado a los objetivos } \\
\text { académicos establecidos en } \\
\text { una determinada actividad } \\
\text { de aprendizaje. }\end{array}$ & $\begin{array}{l}\text { Propuesta dirigida a } \\
\text { formar al estudiante } \\
\text { en el procesamiento de } \\
\text { información, a partir de una } \\
\text { participación activa y sobre } \\
\text { la base de representaciones } \\
\text { mentales que intervienen } \\
\text { en la formación de sus } \\
\text { aprendizajes. }\end{array}$ & $\begin{array}{l}\text { El principal principio de } \\
\text { este enfoque se centra } \\
\text { en la participación del } \\
\text { estudiante en sus procesos } \\
\text { de aprendizaje, con } \\
\text { respaldo en la exploración, } \\
\text { la autoconfianza y la } \\
\text { motivación. }\end{array}$ & $\begin{array}{l}\text { Estudio relativamente } \\
\text { creciente que postula la } \\
\text { extensión del aprendizaje } \\
\text { a entornos virtuales, en } \\
\text { interacción con diversos } \\
\text { recursos tecnológicos y con } \\
\text { el manejo apropiado de } \\
\text { información. }\end{array}$ \\
\hline
\end{tabular}

una tendencia que cobra cada vez más fuerza en el mundo de la enseñanza y el aprendizaje.

\section{LA TAREA INTEGRADA, POR DAVID MERRILL}

Marriner David Merrill es un investigador en el campo educativo, nacido en Norteamérica, quien ha orientado su práctica en los aportes de la tecnología y del diseño instruccional. $\mathrm{Su}$ contribución consiste, principalmente, en postular que la instrucción debe darse de manera organizada a partir del ordenamiento de las acciones o fases de una actividad de aprendizaje. En consecuencia, este principio ubica al docente y al diseño de las rutas de enseñanza que asumirá como ejes importantes de los procesos académicos ejecutados en el aula. En tanto, "las actividades son las acciones y experiencias concretas de enseñanza aprendizaje que el alumno y el profesor realizan día a día en el transcurso de las clases. A través de ellas se trabajan los contenidos para conseguir los objetivos propuestos (...)".10 En ese sentido, una de las actividades que podría considerarse sumamente funcional en este camino es la propuesta de tareas o el trabajo por proyectos, en la medida que facilitan el contacto de información interdisciplinaria, cuya riqueza fortalece la formación de los educandos. A partir de ello, es posible aproximarse a la siguiente afirmación: Una persona es competente cuando es capaz de resolver problemas, aplicando los conocimientos, las capacidades y las conductas pertinentes desarrollados a lo largo de su aprendizaje en la vida.

Lo anterior permite apreciar que este aporte sobre el estudio del aprendizaje debe considerarse como un gran valor en el área de instrucción. En esta línea, Merrill, Tennyson y Posey11 sentencian lo siguiente en su obra Teaching Concepts: "In formal training situations, time is valuable for both the student and the instructor. Since the acquisition of diffciult concepts in a classrroom environments can be time consuming, it is important to use efficient instructionald methods". En términos concretos, tanto el estudiante como el docente valoran la inversión del tiempo en la realización de actividades académicas que conducen al aprendizaje, por lo que resulta tremendamente funcional usar métodos de enseñanza eficientes para concretar objetivos instruccionales.

Este investigador desarrolló cinco principios que orientan los procesos de aprendizaje y el planteamiento de la instrucción. Esta propuesta se respalda en las teorías cognitivista y constructivista, así como en el conectivismo, revisadas en el apartado anterior. En la siguiente figura, se puede apreciar el ciclo de aprendizaje planteado por Merrill12: 


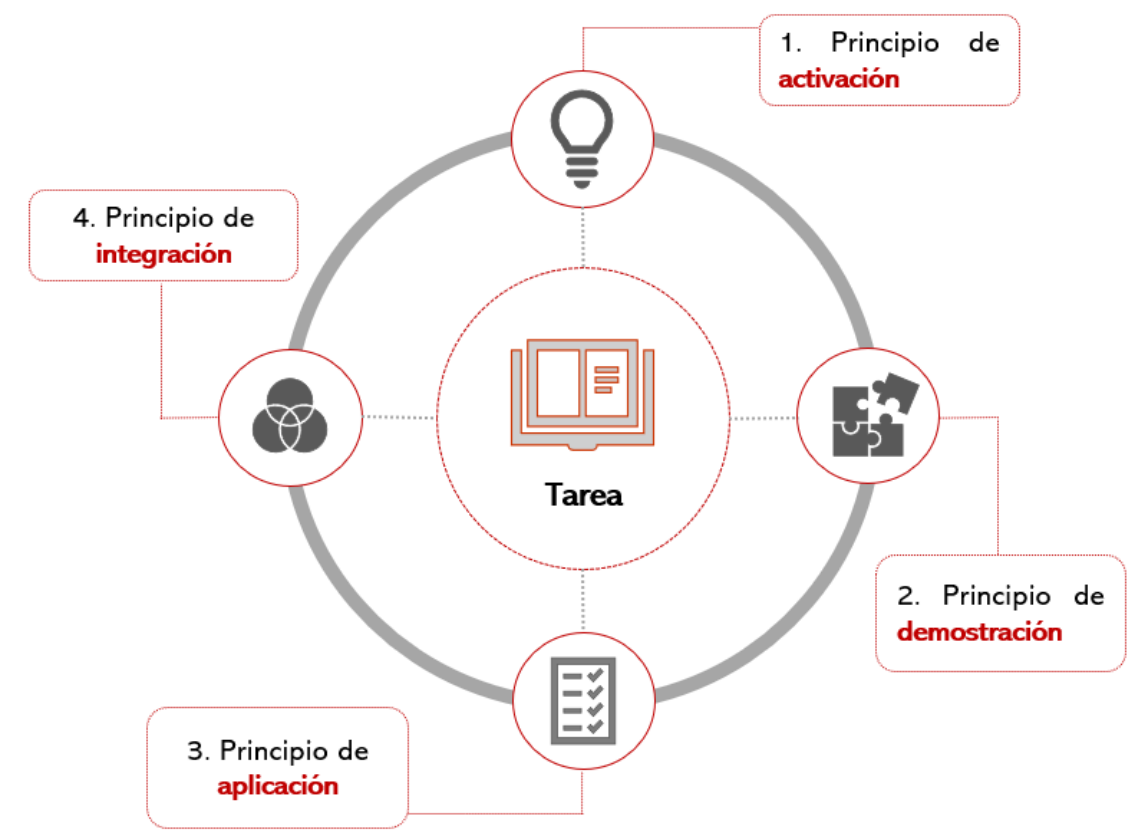

Fuente: La autora.

Figura 1. Etapas del proceso de aprender

\section{ETAPAS DEL PROCESO DE APRENDIZAJE}

En la figura anterior, la tarea (actividad de aprendizaje) se sitúa en el centro mismo del proceso, a partir del planteamiento de situaciones reales. Para cumplir este fin, es importante haber definido de manera preliminar cuál es el objetivo de la tarea y cómo aporta este en la ruta de aprendizaje. Las actividades instruccionales centradas en la tarea, por lo general, instruyen al aprendiz en cada uno de los componentes de la tarea, de lo más simple a lo más complejo (...) hasta que todos sus componentes han sido aprendidos. "Para cada tarea, los aprendices realizan una mayor parte de la misma y reciben menos soporte, hasta que son capaces de llevarla a cabo por sí mismos" (7).

Esta condición se cumplirá progresivamente si se concretan los demás principios, los cuales son aplicables y observables durante el proceso de aprendizaje.

\section{Principio de ACTIVACIÓN}

Desde el planteamiento de la actividad concreta, se valora el intercambio de información, a partir del enfoque del trabajo colaborativo, el cual favorece la formación de nuevos esquemas de contenido. Por ello, se promueve un ambiente que favorece la activación y socialización de experiencias previas relacionadas a la competencia que se pretende desarrollar en el estudiante. Por otro lado, otro de los componentes relevantes en este camino es la construcción de estructuras lógicas, una vez que se adquiere información. Esto consiste, básicamente, en procesar funcionalmente el contenido mediante distintas estrategias, como el sumillado, el resumen, la elaboración de organizadores visuales, entre otras alternativas. 


\section{Principio de Demostración}

Este principio parte de la actividad del docente, quien debe concretar una demostración lo suficientemente consistente para que el estudiante forme una concepción clara sobre la relación entre la habilidad que se pretende consolidar y el tema que se desarrolla en el aula de clase. Así, la demostración "pone énfasis en el uso de casos específicos, así como la representación efectiva y eficiente de la instrucción a través de la información presente y la demostración de esta". ${ }^{\prime}$

Otro componente relevante en esta etapa es la proyección de orientaciones sobre la tarea. Si bien el fin inmediato se relaciona con la exhibición de pautas específicas sobre la actividad en cuestión, debe establecerse una relación notoria con las capacidades generales que se busca desarrollar en los aprendices. Si estos aprecian el impacto a mediano y largo plazo, el aprendizaje será significativo.

\section{Principio de APLicación}

La aplicación es entendida como un proceso en el que se ejecutan (o usan) los conocimientos y las habilidades en proceso de adquisición para solucionar un problema o una situación propuesta, en atención a lo observado en la etapa de demostración. En este camino, la retroalimentación cumpleun papel interviniente fundamental, siempre que el estudiante puede evaluar sus decisiones de selección y reflexionar sobre las consecuencias inmediatas. También, es posible que aprecie las formas de optimizar su desempeño en consonancia con lo expuesto en la demostración. Finalmente, otro elemento favorecedor en esta etapa es la colaboración entre pares, por cuanto el aprendiz participa en una actividad y tiene la posibilidad de intercambiar pareceres, propuestas y por qué no, proponer consensos a sus compañeros.

\section{Principio de integración}

El principio de integración se materializa cuando los estudiantes toman los conocimientos recientemente adquiridos y los ponen en práctica en situaciones cotidianas de su vida. Este no es un proceso simple, pues está permanentemente acompañado de periodos de reflexión, cuyas acciones se centran en la formulación de críticas constructivas entre pares, en la discusión y defensa de posturas, así como en la emisión de propuestas para optimizar la tarea. Por otra parte, el cuestionamiento entre pares ${ }^{12}$ también favorece el aprendizaje, en la medida que amplía la perspectiva sobre el desarrollo de la actividad y determina criterios de comparación con el desempeño de sus pares. En ese mismo camino, las demostraciones públicas se orientan a fortalecer las habilidades comunicativas para transmitir, explicar y defender su propuesta frente a posibles críticas por parte de sus compañeros.

\section{EL USO DE WIKIS COLABORATIVAS COMO ALTERNATIVA} PARA LA APLICACIÓN DE LA TAREA INTEGRADA

En este apartado, se hace principal referencia a una de las tantas herramientas tecnológicas que dan soporte actualmente a los procesos de aprendizaje. Como bien se sabe, hoy en día muchas instituciones, entre públicas y privadas, de educación básica y superior, utilizan repositorios virtuales a los que acceden administradores, estudiantes $y$ docentes. A pesar de que la participación de los agentes mencionados difiere en el grado de intervención y protagonismo en su uso, se menciona algunas de las actividades y recursos con presencia notoria en este tipo de espacio virtual:

Una aclaración necesaria en este punto es delimitar las diferencias entre los términos 
Tabla 2: Recursos y actividades en plataformas virtuales de aprendizaje

\begin{tabular}{ll}
\hline Recursos & Actividades \\
\hline Archivo & Cuestionario \\
Etiqueta & Foro \\
Página & Tarea \\
URL & Wiki \\
Módulo de aprendizaje & Blog \\
Paquete SCORM & Grupos \\
\hline
\end{tabular}

recursos y actividades en entornos virtuales de aprendizaje. Por una parte, los recursos son elementos de gran utilidad para proveer información a una comunidad virtual. En otros términos, los docentes utilizan estas herramientas para compartir información de interés para los aprendizajes, contenidos que se encuentran alineados a los objetivos de aprendizaje propuestos en el curso. Por otro lado, las actividades se concretan en tareas que demandan el uso de la información anterior para promover el desarrollo de determinadas habilidades. A través de dichas actividades, los estudiantes ejercitan lo aprendido y hacen uso de todos los recursos con los que cuentan para culminar con éxito la tarea encargada.

Para la presentación de esta propuesta, se ha considerado las coincidencias en cuanto a los recursos y las herramientas utilizados en dos de las plataformas más conocidas en el ámbito de la educación superior: Moodle y Blackboard, en sus versiones básicas. De aquí en adelante, se presenta una breve reseña de la herramienta wiki, una de las menos localizadas en entornos virtuales académicos y que posee características de alto potencial propicias para fortalecer el aprendizaje de los estudiantes, así como para favorecer la labor de enseñanza de los educadores.

Las wikis son herramientas eficaces capaces de articular y favorecer el desarrollo de proyectos colaborativos, debido a su naturaleza abierta y flexible, que a través de una interfaz sencilla y amigable posibilita la interacción y comunicación entre los estudiantes que forman un determinado grupo de trabajo, facilitando el proceso de construcción colectiva del conocimiento. ${ }^{13}$

Al hacer referencia a sus principales características, es posible mencionar las siguientes, según lo expuesto por Araujo: ${ }^{14}$

- Los usuarios pueden ser autores, editores y colaboradores, en lugar de lectores o "consumidores de información".

- Las wikis son espacios amigables, de fácil uso, que contribuyen a la autoría colectiva.

- La información puede editarse de forma permanente, siempre en condición asincrónica.

- A nivel de evaluación/valoración de desempeños, las wikis permiten apreciar el proceso de producción y medir, cuantitativa y cualitativamente, el nivel de aportación de cada integrante en un equipo de trabajo.

El modelo de la tarea integrada aplicado en wikis colaborativas es una alternativa de trabajo en el aula de clase, que incorpora el aporte de Merrill, desde la perspectiva del aprendizaje mediado por la tecnología, en el uso de recursos y actividades virtuales funcionales para los procesos de enseñanzaaprendizaje. En ese sentido, la wiki es el espacio en que el estudiante inicia la tarea, la cual será regulada por los principios de la tarea integrada revisados en este artículo. La siguiente figura propone una primera propuesta de trabajo, a partir de la consigna de un proyecto colaborativo de escritura:

Si bien es cierto, el avance de este proceso implica el incremento de la complejidad en la realización de la actividad, la intervención y el acompañamiento del docente son cruciales. 
Tarea central: Redacción colaborativa de un texto en una wiki de trabajo (Aula virtual)

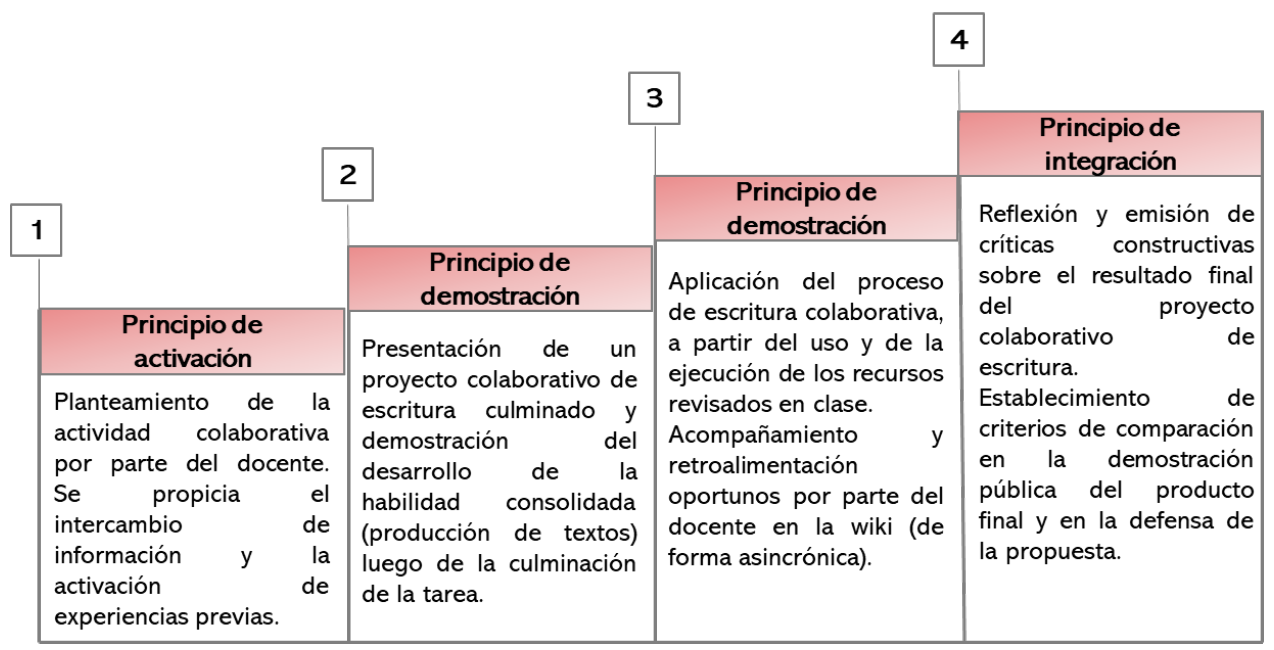

Fuente: Elaboración propia

Figura 2. Tarea integrada en una wiki colaborativa

De hecho, el seguimiento del profesor debería disminuir a medida que el estudiante se involucre más con la tarea y con los objetivos de esta. Adicionalmente, es importante destacar que la retroalimentación se condensa de forma transversal en la implementación de este modelo instruccional.

En suma, los principios estudiados en la sección anterior pueden materializarse en la colaboración virtual de estudiantes en plataformas digitales educativas en consonancia y aprovechamiento directo de las características de esta tarea/actividad virtual.

\section{REFERENCIAS BIBLIOGRÁFICAS}

1. Hernández G. Módulo Fundamentos del Desarrollo de la Tecnología Educativa (Bases Psicopedagógicas). Paidós; 1997.

2. Rodríguez P. Revisión de las teorías del aprendizaje más sobresalientes del siglo XX. Tiempo Educ. 2004;5(10):39-76.

3. López M, López A. Los enfoques de aprendizaje. Revisión conceptual y de investigación. Rev Colomb Educ. 2013;64:13153.

4. Leiva C. Conductismo, cognitivismo y aprendizaje. Tecnol en Marcha. 2005;18(1):66-73.

5. Ovelar R, Benito M, Romo J. Nativos digitales y aprendizaje. Una aproximación a la evolución de este concepto. ICONO 14. 2009;7(1):31-53.

6. Hernández G. Paradigmas de la psicología de la educación. Paidós; 1998.
7. Del Valle R, Tueros E. Fundamentos psicopedagógicos y éticos del aprendizaje adulto. Facultad de Eduación - Pontifica Universidad Católica del Perú; 2017. 115 p.

8. Zapata M. Teorías y modelos sobre sobre el aprendizaje en entornos conectados y ubicuos. Bases para un nuevo modelo teórico a partir de una visión crítica del "conectivismo." Educ Knowl Soc. 2015;16(1):69-102.

9. Díaz Á. El enfoque de competencias en la educación. ¿Una alternativa o un disfraz de cambio? Perfiles Educ. 2006;28(111):736.

10. Quevedo-Blasco R, Quevedo-Blasco V, Téllez M, Buela-Casal G. La tarea integrada como herramienta para mejorar los procesos cognitivos en tareas de aprendizaje. En: Situación Actual de la Psicología Clínica [Internet]. 2011. p. 467. URL disponible en: https://www.ugr.es/ aepc/WEBCLINICA/ DOCUMENTOS / librocapitulosIX.pdf\# page $=84$

11. Merrill D, Tennyson R, Posey L. Teaching Concepts [Internet]. 1992. 218 p. URL disponible en: https:// books.google.es / books?hl=es\&lr=\&id=MEg syt6jyzHIKXjqatXsc\# $\mathrm{v}=$ onepage $\& \mathrm{q}=$ david merrill\& $\mathrm{f}=$ false

12. Reigeluth $\mathrm{C}$. Teoría instruccional y tecnología para el nuevo paradigma de la educación. Rev Educ a Distancia. 2011;32:1-22.

13. Del Moral M, Villalustre L. Las wikis vertebradoras del trabajo colaborativo universitario a través del WebQuest. Rev Latinoam Tecnol Educ. 2008;7(1):73-83.

14. Araujo J. El uso de blogs, wikis y redes sociales en la enseñanza de lenguas. EDUTEC. Rev Electrónica Tecnol Educ. 2014;49:1-27.

\section{CORRESPONDENCIA:}

Rossana Fiorella Felipa-Andrade, rossana.felipa@upn.edu.pe

Fecha de recepción: 03-03-2020

Fecha de aceptación: 22-06-2020. 